\title{
Clamped cylindrical shells of laminated composites: effect of cutout on natural frequency and mode shapes
}

\begin{abstract}
Clamped cylindrical shells made of laminated composite materials in presence of stiffeners and cutouts are analyzed employing the eight-noded curved quadratic isoperimetric element for shell with a three noded beam element for stiffener formulation. Free vibration problem of stiffened cylindrical shells with different size and position of the cutouts with respect to the shell centre are examined to find natural frequency and mode shapes of stiffened shells and arrive at some conclusions useful to the designers. The results are further analyzed to suggest guidelines to select optimum size and position of the cutout with respect to shell centre.
\end{abstract}

Keywords: clamped cylindrical shell, laminated composite, cutout, natural frequency, mode shape
Volume 2 Issue 4 - 2017

\author{
Sarmila Sahoo \\ Department of Civil Engineering, Heritage Institute of \\ Technology, India
}

\begin{abstract}
Correspondence: Sarmila Sahoo, Department of Civil Engineering, Heritage Institute of Technology, Kolkata 700107,
\end{abstract} India, Email sarmila.sahoo@gmail.com

Received: February 16, 2017| Published: April 18, 2017

\section{Introduction}

Laminated composites are increasingly being used nowadays in aerospace, civil, marine and other related weight-sensitive engineering applications requiring high strength-to-weight and stiffness-to weight ratios. Cylindrical shells, a special type of shells, are found in many applications in the aerospace and naval construction industries. They are often used as load-bearing structures for aircrafts, rockets, submarines and missile bodies. Of course, the cylindrical shells used in those applications are designed with stiffeners to provide better strength, stiffness and buckling characteristics. Cutouts are provided in shell panels to save weight and also to provide a facility for inspection. In practice the margin of the cutouts are stiffened to take account of stress concentration effects. Also, there can be some instruments directly fixed on these panels, and the safety of these instruments can be dependent on the vibration characteristics of the panels. Hence free vibration studies on cylindrical shell panels with cutouts are of interest to structural engineers.

Dynamic analysis of shell structures having complex geometry, loading and boundary conditions can be solved efficiently by finite element method. Different computational models for laminated composites were proposed by researchers. Vibration of shells is an extensively studied area in mechanical and structural dynamics. The natural frequencies of the cylindrical shells lie in a narrow band and they are prone to becoming involved in resonant vibrations. To control the amplitudes of these vibrations, it is necessary to know the distribution of the natural frequencies in order to design the cylindrical shell structures safely from the viewpoint of optimum vibration control. ${ }^{1}$ Vibration of cylindrical shells has been extensively studied by many researchers. ${ }^{2,3}$ Warburton ${ }^{4}$ contributed information about the vibration of thin cylindrical shells. Leissa et al. ${ }^{5} \&$ Chung $^{6}$ studied the free vibration characteristics of cylindrical shells. Free vibration of antisymmetric angle-ply laminated circular cylindrical panels was studied by Soldatos. ${ }^{7}$ A review on vibration of isotropic and multilayered laminated cylindrical shells is also available. ${ }^{8}$ Leissa ${ }^{9}$ presented a detailed discussion on shell vibration problems and provides different aspects of vibration of cylindrical shells with different shell theories and boundary conditions. A bulk of research papers are found on the vibration of cylindrical shells without cutout. Dennis \& Palazotto ${ }^{10}$ and Nanda \& Bandyopadhyay ${ }^{11}$ concentrated on static and dynamic responses respectively of a cylindrical composite panel with cutout using a geometrically nonlinear theory. However natural frequency and vibration mode shapes of clamped cylindrical shells with cutout is not present in the existing body of literature. Accordingly, the present study considers natural frequencies and mode shape of composite cylindrical shell with cutout (stiffened along the margin) with concentric and eccentric cutouts, and considers the shell panel to be clamped at all edges.

\section{Formulation}

A laminated composite cylindrical shell of uniform thickness $h$ (Figure 1) and radius of curvature $R_{y}$ is considered. Keeping the total thickness same, the thickness may consist of any number of thin laminate each of which may be arbitrarily oriented at an angle $\theta$ with reference to the $X$-axis of the co-ordinate system. The constitutive equations for the shell are given by

$$
\{F\}=[E]\{\varepsilon\}
$$

Where,

$$
\begin{aligned}
& \{F\}=\left\{N_{x}, \quad N_{y}, \quad N_{x y}, M_{x}, M_{y}, M_{x y}, Q_{x}, Q_{y}\right\}^{T} \text {, } \\
& {[E]=\left[\begin{array}{lll}
{[A]} & {[B]} & {[0]} \\
{[B]} & {[D]} & {[0]} \\
{[0]} & {[0]} & {[S]}
\end{array}\right],}
\end{aligned}
$$

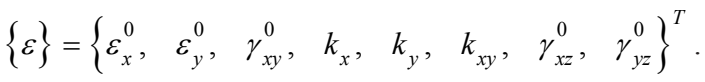

The force and moment resultants are expressed as

$$
\begin{aligned}
& \left\{N_{x}, N_{y}, N_{x y}, M_{x}, M_{y}, M_{x y}, Q_{x}, Q_{y}\right\}^{T} \\
& =\int_{-h / 2}^{h / 2}\left\{\sigma_{x}, \quad \sigma_{y}, \quad \tau_{x y}, \quad \sigma_{z} . z, \quad \sigma_{y} . z, \tau_{x y} . z, \tau_{x z}, \tau_{y z}\right\}^{T} d z
\end{aligned}
$$


The sub matrices $[A],[B],[D]$ and $[S]$ of the elasticity matrix $[E]$ are functions of Young's moduli, shear moduli and the Poisson's ratio of the laminates. They also depend on the angle which the individual lamina of a laminate makes with the global $X$-axis. The

$$
\left\{\varepsilon_{x}, \quad \varepsilon_{y}, \quad \gamma_{y}, \quad \gamma_{x}, \quad \gamma_{y}\right\}^{T}=\left\{\varepsilon_{x}^{0}, \quad \varepsilon_{y}^{0}, \quad \gamma_{y}^{0}, \quad \gamma_{x}^{0},\right.
$$

Where, the first vector is the mid-surface strain for a cylindrical shell and the second vector is the curvature.

An eight-noded curved quadratic isoperimetric finite element is used for cylindrical shell analysis. The five degrees of freedom taken into consideration at each node are $u, v, w, \beta$. The following expressions establish the relations between the displacement at any point with respect to the co-ordinates $\xi$ and $\eta$ and the nodal degrees of freedom.

$u=\sum_{i=1}^{8} N_{i} u_{i} \quad v=\sum_{i=1}^{8} N_{i} v_{i} \quad w=\sum_{i=1}^{8} N_{i} w_{i} \quad \alpha=\sum_{i=1}^{8} N_{i} \alpha_{i} \quad \beta=\sum_{i=1}^{8} N_{i} \beta_{i}$

where the shape functions derived from a cubic interpolation polynomial $^{23}$ are:

$$
\begin{aligned}
& N_{i}=\left(1+\xi \xi_{i}\right)\left(1+\eta \eta_{i}\right)\left(\xi \xi_{i}+\eta \eta_{i}-1\right) / 4 \text { for } i=1,2,3,4 \\
& N_{i}=\left(1+\xi \xi_{i}\right)\left(1-\eta^{2}\right) / 2 \text { for } i=5,7 \\
& N i=\left(1+\eta \eta_{i}\right)\left(1-x^{2}\right) / 2, \text { for } i=6,8
\end{aligned}
$$

The generalized displacement vector of an element is expressed in terms of the shape functions and nodal degrees of freedom as:

$$
[u]=[N]\left\{d_{e}\right\}
$$

$$
\text { i.e., }\{u\}=\left\{\begin{array}{c}
u \\
v \\
w \\
\alpha \\
\beta
\end{array}\right\}=\sum_{i=1}^{8}\left[\begin{array}{ccccc}
N_{i} & & & & \\
& N_{i} & & & \\
& & N_{i} & & \\
& & & N_{i} & \\
& & & & N_{i}
\end{array}\right]\left\{\begin{array}{c}
u_{i} \\
v_{i} \\
w_{i} \\
\alpha_{i} \\
\beta_{i}
\end{array}\right\}
$$

The strain-displacement relation is given by

$\{\varepsilon\}=[B]\left\{d_{e}\right\}$,

Where $[B]=\sum_{i=1}^{8}\left[\begin{array}{ccccc}N_{i, x} & 0 & 0 & 0 & 0 \\ 0 & N_{i, y} & -\frac{N_{i}}{R_{y}} & 0 & 0 \\ N_{i, y} & N_{i, x} & 0 & 0 & 0 \\ 0 & 0 & 0 & N_{i, x} & 0 \\ 0 & 0 & 0 & 0 & N_{i, y} \\ 0 & 0 & 0 & N_{i, y} & N_{i, x} \\ 0 & 0 & N_{i, x} & N_{i} & 0 \\ 0 & 0 & N_{i, y} & 0 & N_{i}\end{array}\right]$

The element stiffness matrix is

$$
\left[K_{e}\right]=\iint[B]^{T}[E][B] d x d y
$$

The element mass matrix is obtained from the integral detailed expressions of the elements of the elasticity matrix are available in several references including Vasiliev et al. ${ }^{12}$ \& Qatu. ${ }^{13}$ The strain-displacement relations on the basis of improved first order approximation theory for thin shell are established as

$\left.\gamma_{y}^{0}\right\}^{T}+z\left\{k_{x}, \quad k_{y}, \quad k_{y}, \quad k_{x}, k_{y}\right\}^{T}$

$$
\left[M_{e}\right]=\iint[N]^{T}[P][N] d x d y,
$$

where,

$[N]=\sum_{i=1}^{8}\left[\begin{array}{ccccc}N_{i} & 0 & 0 & 0 & 0 \\ 0 & N_{i} & 0 & 0 & 0 \\ 0 & 0 & N_{i} & 0 & 0 \\ 0 & 0 & 0 & N_{i} & 0 \\ 0 & 0 & 0 & 0 & N_{i}\end{array}\right],[P]=\sum_{i=1}^{8}\left[\begin{array}{ccccc}P & 0 & 0 & 0 & 0 \\ 0 & P & 0 & 0 & 0 \\ 0 & 0 & P & 0 & 0 \\ 0 & 0 & 0 & I & 0 \\ 0 & 0 & 0 & 0 & I\end{array}\right]$,

in which

$$
P=\sum_{k=1}^{n p} \int_{z_{k-1}}^{z_{k}} \rho d z \text { and } I=\sum_{k=1}^{n p} \int_{z_{k-1}}^{z_{k}} z \rho d z
$$

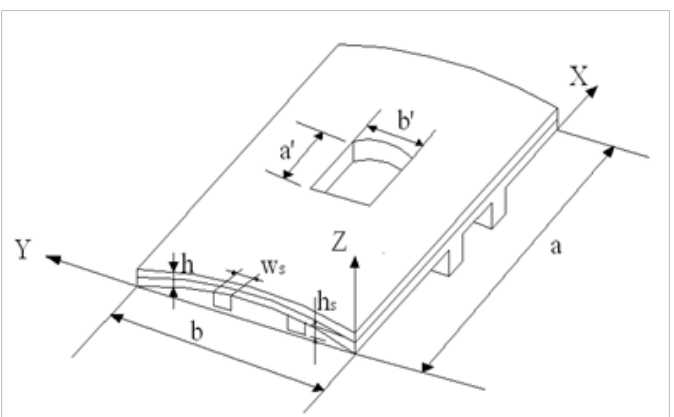

Figure I Cylindrical shell with a concentric cutout stiffened along the margins.

Three noded curved isoperimetric beam elements are used to model the stiffeners, which are taken to run only along the boundaries of the shell elements. In the stiffener element, each node has four degrees of freedom i.e. $u_{s x}, w_{s x}, \alpha_{s x}$ and $\beta_{s x}$ for $X$-stiffener and $v_{s y}, w_{s y}, \alpha_{s y}$ and $\beta_{s y}$ for $Y$-stiffener. The generalized force-displacement relation of stiffeners can be expressed as:

$$
\begin{aligned}
& \text { X-stiffener: }\left\{F_{s x}\right\}=\left[D_{s x}\right]\left\{\varepsilon_{s x}\right\}=\left[D_{s x}\right]\left[B_{s x}\right]\left\{\delta_{s x i}\right\} ; \\
& \text { Y-stiffener: }\left\{F_{s y}\right\}=\left[D_{s y}\right]\left\{\varepsilon_{s y}\right\}=\left[D_{s y}\right]\left[B_{s y}\right]\left\{\delta_{s y i}\right\}
\end{aligned}
$$

where, $\left\{F_{s x}\right\}=\left[\begin{array}{llll}N_{s x x} & M_{s x x} & T_{s x x} & Q_{s x x z}\end{array}\right]^{T}$;

$$
\begin{gathered}
\left\{\varepsilon_{s x}\right\}=\left[\begin{array}{llll}
u_{s x . x} & \alpha_{s x . x} & \beta_{s x . x} & \left(\alpha_{s x}+w_{s x . x}\right)
\end{array}\right]^{T} \\
\text { and }\left\{F_{s y}\right\}=\left[\begin{array}{llll}
N_{s y y} & M_{s y y} & T_{s y y} & Q_{s y y z}
\end{array}\right]^{T} ; \\
\left\{\varepsilon_{s y}\right\}=\left[\begin{array}{llll}
v_{s y . y} & \beta_{s y . y} & \alpha_{s y . y} & \left(\beta_{s y}+w_{s y . y}\right)
\end{array}\right]^{T}
\end{gathered}
$$

The generalized displacements of the $y$-stiffener and the shell are related by the transformation matrix $\left\{\delta_{s y i}\right\}=[T]\{\delta\}$ where 


$$
[T]=\left[\begin{array}{ccccc}
1+\frac{e}{R_{y}} & \text { symmetric } & & \\
0 & 1 & & \\
0 & 0 & 1 & \\
0 & 0 & 0 & 1
\end{array}\right]
$$

This transformation is required due to curvature of $y$-stiffener and $\{\delta\}$ is the appropriate portion of the displacement vector of the shell excluding the displacement component along the x-axis.

Elasticity matrices are as follows:

$$
\begin{aligned}
& {\left[D_{s x}\right]=\left[\begin{array}{cccc}
A_{11} b_{s x} & B^{\prime}{ }_{11} b_{s x} & B^{\prime}{ }_{12} b_{s x} & 0 \\
B^{\prime}{ }_{11} b_{s x} & D^{\prime}{ }_{11} b_{s x} & D^{\prime}{ }_{12} b_{s x} & 0 \\
B^{\prime}{ }_{12} b_{s x} & D^{\prime}{ }_{12} b_{s x} & \frac{1}{6}\left(Q_{44}+Q_{66}\right) d_{s x} b_{s x}^{3} & 0 \\
0 & 0 & 0 & b_{s x} S_{11}
\end{array}\right]} \\
& {\left[D_{s y}\right]=\left[\begin{array}{cccc}
A_{22} b_{s y} & B^{\prime}{ }_{22} b_{s y} & B^{\prime}{ }_{12} b_{s y} & 0 \\
B^{\prime}{ }_{22} b_{s y} & \frac{1}{6}\left(Q_{44}+Q_{66}\right) b_{s y} & D^{\prime}{ }_{12} b_{s y} & 0 \\
B_{12}^{\prime}{ }_{12} b_{s y} & D^{\prime}{ }_{12} b_{s y} & D^{\prime}{ }_{11} d_{s y} b_{s y}^{3} & 0 \\
0 & 0 & 0 & b_{s y} S_{22}
\end{array}\right] .}
\end{aligned}
$$

where,

$$
D_{i j}^{\prime}=D_{i j}+2 e B_{i j}+e^{2} A_{i j} ; B_{i j}^{\prime}=B_{i j}+e A_{i j},
$$

and $A_{i j}, B_{i j}, D_{i j}$ and $S_{i j}$ are explained in an earlier paper by Sahoo \& Chakravorty. ${ }^{14}$

Here the shear correction factor is taken as 5/6. The sectional parameters are calculated with respect to the mid-surface of the shell by which the effect of eccentricities of stiffeners is automatically included. The element stiffness matrices are of the following forms.

$$
\begin{aligned}
& \text { For X-stiffener: }\left[K_{x e}\right]=\int\left[B_{s x}\right]^{T}\left[D_{s x}\right]\left[B_{s x}\right] d x \\
& \text { for Y-stiffener: }\left[K_{y e}\right]=\int\left[B_{s y}\right]^{T}\left[D_{s y}\right]\left[B_{s y}\right] d y
\end{aligned}
$$

The integrals are converted to isoperimetric coordinates and are carried out by 2-point Gauss quadrature. Finally, the element stiffness matrix of the stiffened shell is obtained by appropriate matching of the nodes of the stiffener and shell elements through the connectivity matrix and is given as:

$$
\left[K_{e}\right]=\left[K_{s h e}\right]+\left[K_{x e}\right]+\left[K_{y e}\right] \text {. }
$$

The element stiffness matrices are assembled to get the global matrices.

The element mass matrix for shell is obtained from the integral

$$
\left[M_{e}\right]=\iint[N]^{T}[P][N] d x d y,
$$

Where,

$$
[N]=\sum_{i=1}^{8}\left[\begin{array}{ccccc}
N_{i} & 0 & 0 & 0 & 0 \\
0 & N_{i} & 0 & 0 & 0 \\
0 & 0 & N_{i} & 0 & 0 \\
0 & 0 & 0 & N_{i} & 0 \\
0 & 0 & 0 & 0 & N_{i}
\end{array}\right],[P]=\sum_{i=1}^{8}\left[\begin{array}{ccccc}
P & 0 & 0 & 0 & 0 \\
0 & P & 0 & 0 & 0 \\
0 & 0 & P & 0 & 0 \\
0 & 0 & 0 & I & 0 \\
0 & 0 & 0 & 0 & I
\end{array}\right],
$$

in which

$P=\sum_{k=1}^{n p} \int_{z_{k-1}}^{z_{k}} \rho d z$ and $I=\sum_{k=1}^{n p} \int_{z_{k-1}}^{z_{k}} z \rho d z$

Element mass matrix for stiffener element

$\left[M_{s x}\right]=\iint[N]^{T}[P][N] d x \quad$ for X stiffener

and $\left[M_{s y}\right]=\iint[N]^{T}[P][N] d y \quad$ for Y stiffener

Here, $\quad[N]$ is a $3 \times 3$ diagonal matrix.

$[P]=\sum_{i=1}^{3}\left[\begin{array}{cccc}\rho . b_{s x} d_{s x} & 0 & 0 & 0 \\ 0 & \rho . b_{s x} d_{s x} & 0 & 0 \\ 0 & 0 & \rho . b_{s x} d_{s x}^{2} / 12 & 0 \\ 0 & 0 & 0 & \rho\left(b_{s x} \cdot d_{s x}^{3}+b_{s x}^{3} \cdot d_{s x}\right) / 12\end{array}\right]$

For X-stiffener

$[P]=\sum_{i=1}^{3}\left[\begin{array}{cccc}\rho \cdot b_{s y} d_{s y} & 0 & 0 & 0 \\ 0 & \rho \cdot b_{s y} d_{s y} & 0 & 0 \\ 0 & 0 & \rho \cdot b_{s y} d_{s y}^{2} / 12 & 0 \\ 0 & 0 & 0 & \rho\left(b_{s y} \cdot d_{s y}^{3}+b_{s y}^{3} \cdot d_{s y}\right) / 12\end{array}\right]$

\section{for Y-stiffener}

The mass matrix of the stiffened shell element is the sum of the matrices of the shell and the stiffeners matched at the appropriate nodes.

$$
\left[M_{e}\right]=\left[M_{s h e}\right]+\left[M_{x e}\right]+\left[M_{y e}\right] .
$$

The element mass matrices are assembled to get the global matrices. The code developed can take the position and size of cutout as input. The program is capable of generating non uniform finite element mesh all over the shell surface. So the element size is gradually decreased near the cutout margins.

The free vibration analysis involves determination of natural frequencies from the condition

$$
\left|[K]-\omega^{2}[M]\right|=0
$$

This is a generalized Eigen value problem and is solved by the subspace iteration algorithm.

\section{Results and discussion}

The results of Table 1 show that the agreement of present results with the earlier ones is excellent and the correctness of the stiffener 
formulation is established. Free vibration of clamped cylindrical shell of $(0 / 90)_{4}$ lamination with cutouts is also considered. The fundamental frequencies of cylindrical shell with cutout obtained by the present method agree well with those reported by Chakravorty et al. ${ }^{15}$ as evident from Table 1, establishing the correctness of the cutout formulation. Thus it is evident that the finite element model proposed here can successfully analyze vibration problems of stiffened cylindrical composite shells with cutout which is reflected by close agreement of present results with benchmark ones.

$$
/ b=1, a / h=100, a^{\prime} / b=1, h / R_{x x}=0, h / R_{y y}=1 / 300
$$

In order to study the effect of cutout size and position on the free vibration response additional problems for clamped cylindrical shells with 0/90/0/90 and $+45 /-45 /+45 /-45$ laminations have been solved. The positions of the cutouts are varied along both of the plan directions of the shell to study the effect of eccentricity of cutout on the fundamental frequency.

Table I Non-dimensional fundamental frequencies $(\bar{\omega})$ for clamped laminated composite cylindrical shell with cutout

\begin{tabular}{lll}
\hline a'/a $^{\prime}$ & Chakravorty et al. ${ }^{15}$ & Present model \\
\hline 0 & 69.36 & 69.02 \\
0.1 & 68.776 & 68.66 \\
0.2 & 64.751 & 64.23 \\
0.3 & 59.317 & 58.83 \\
0.4 & 59.303 & 59.11 \\
\hline
\end{tabular}

Table 2 furnishes the results of non-dimensional frequency $(\bar{\omega}$ ) of 0/90/0/90 and $+45 /-45 /+45 /-45$ stiffened cylindrical shells with cutout. The shells considered are of square plan form $(\mathrm{a}=\mathrm{b})$ and the cutouts are also taken to be square in plan $\left(a^{\prime}=b^{\prime}\right)$. The cutouts placed concentrically on the shell surface. The cutout sizes (i.e. a/a) are varied from 0 to 0.4 . The stiffeners are place along the cutout periphery and extended up to the edge of the shell. The material and geometric properties of shells and cutouts are mentioned along with the Table.

$$
\begin{gathered}
a / b=1, a / h=100, a^{\prime} / b^{\prime}=1, h / R_{x x}=0, h / R_{y y}=1 / 300 ; E_{11} / E_{22}=25, G_{23} \\
=0.2 E_{22}, G_{13}=G_{12}=0.5 E_{22}, v_{12}=v_{21}=0.25 .
\end{gathered}
$$

Table 2 Non-dimensional fundamental frequencies $(\omega)$ for laminated composite stiffened cylindrical shell for different sizes of the central square cutout and different laminations

\begin{tabular}{llllll} 
Laminations & \multicolumn{6}{l}{ Cutout size $\left(a^{\prime} / a\right)$} \\
\hline $0 / 90 / 0 / 90$ & $\mathbf{0}$ & $\mathbf{0 . 1}$ & $\mathbf{0 . 2}$ & $\mathbf{0 . 3}$ & $\mathbf{0 . 4}$ \\
0.00049383 & 91.97 & 103.45 & 111.3 & 110.68 & 110.34 \\
\hline
\end{tabular}

From Table 2 it is seen that when a cutout is introduced to a stiffened shell the fundamental frequency changes in all the cases. This trend is noticed for both cross ply and angle ply shells. This initial increase in frequency is due to the fact that with the introduction of cutout, numbers of stiffeners increases from two to four in the present study. As the cutout grows in size the loss of mass is more significant than loss of stiffness, and hence the frequency increases. As with the introduction of a cutout of $\mathrm{a}^{\prime} / \mathrm{a}=0.2$, in shell surface, the frequency increases in most of the cases, this leads to the engineering conclusion that concentric cutouts with stiffened margins may be provided safely on shell surfaces for functional requirements upto $\mathrm{a} / \mathrm{a}=0.2$.

The mode shapes corresponding to the fundamental modes of vibration are plotted in Figure 2,3 for cross ply and angle ply shells respectively. The normalized displacements are drawn with the shell mid-surface as the reference for all the support condition and for all the lamination used here. For corner point supported shells the fundamental mode shapes are complicated. With the introduction of cutout mode shapes remain almost similar. When the size of the cutout is increased from 0.2 to 0.4 the fundamental modes of vibration do not change to an appreciable amount.

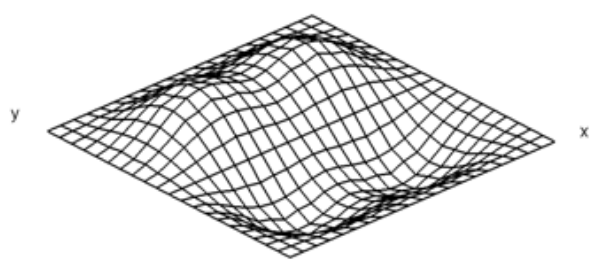

(a)

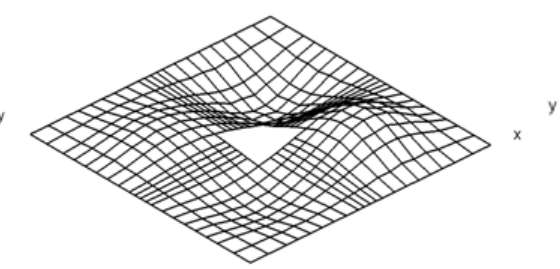

(b)

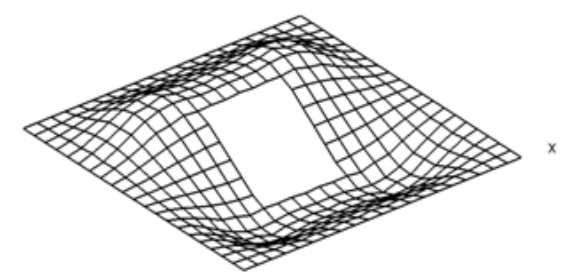

(c)

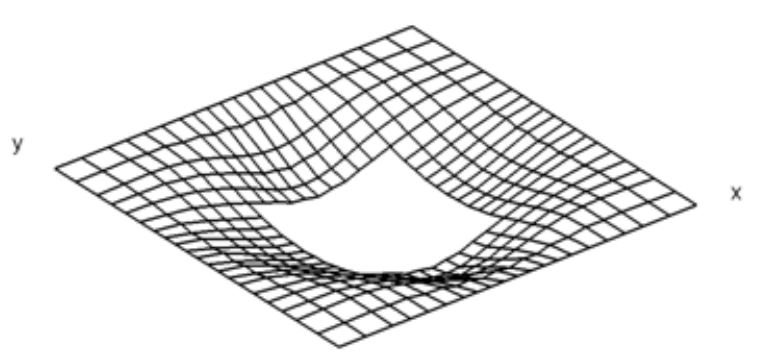

(d)

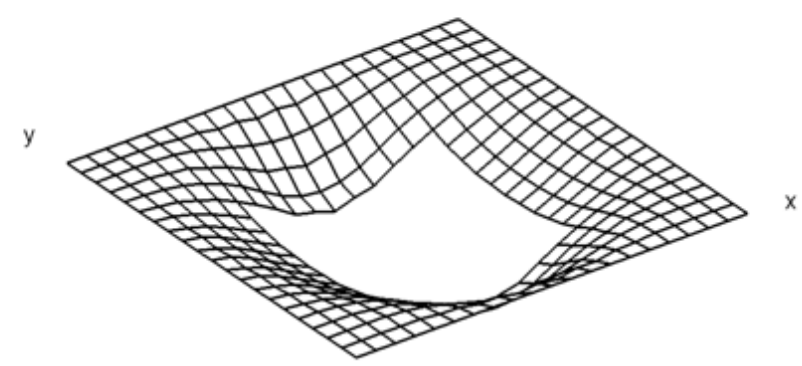

(e)

Figure 2 First mode shapes of laminated composite (0/90/0/90) stiffened cylindrical shell for different sizes of the central square cutout (a) $a^{\prime} / a=0$, (b) $a^{\prime} / a$ $=0.1$, (c) $a^{\prime} / a=0.2$, (d) $a^{\prime} / a=0.3$, (e) $a^{\prime} / a=0.4$. 


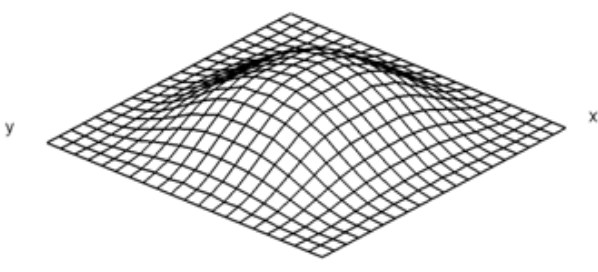

(a)

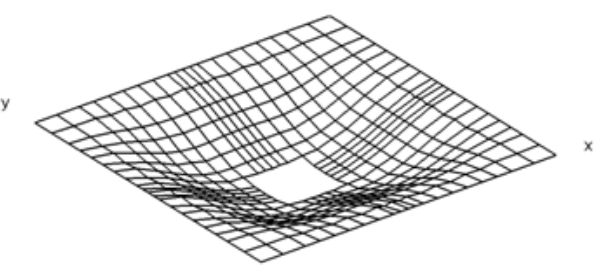

(b)

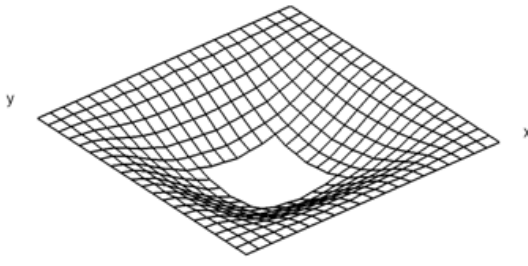

(c)

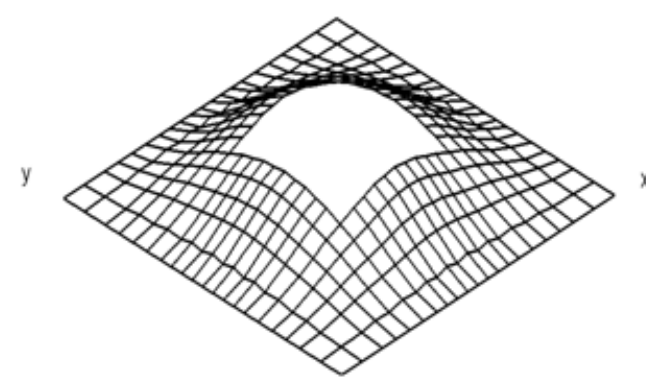

(d)

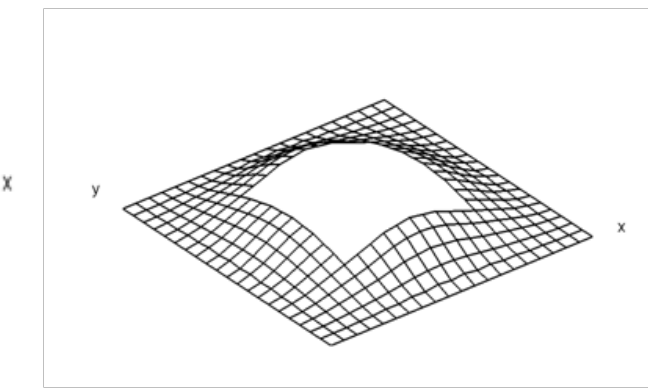

(e)

Figure 3 First mode shapes of laminated composite $(+45 /-45 /+45 /-45)$ stiffened cylindrical shell for different sizes of the central square cutout (a) $a^{\prime} / a=0$, (b) $a^{\prime} / a=0.1$, (c) $a^{\prime} / a=0.2$, (d) $a^{\prime} / a=0.3$, (e) $a^{\prime} / a=0.4$.

The effect of eccentricity of cutout positions on fundamental frequencies, are studied from the results obtained for different locations of a cutout with $a^{\prime} / a=0.2$. The non-dimensional coordinates of the cutout centre $\left(\bar{x}=\frac{x}{a}, \bar{y}=\frac{y}{a}\right)$ was varied from 0.2 to 0.8 along each directions, so that the distance of a cutout margin from the shell boundary was not less than one tenth of the plan dimension of the shell. The fundamental frequency of a shell with an eccentric cutout is expressed as a percentage of fundamental frequency of a shell with a concentric cutout. This percentage is denoted by $r$ in Table 3 . It can be seen that eccentricity of the cutout along the length of the shell towards the edges makes it more flexible. It is also seen that almost all the cases $r$ value is maximum in and around $x=0.5$ and $y=0.5$. It is noticed that for clamped cylindrical shells the maximum fundamental frequency always occurs along the diagonal of the shell. This table indicates the maximum eccentricity of a cutout which can be permitted if the fundamental frequency of a concentrically punctured shell is not to reduce a drastic amount.

Table 3 Values of ' $r$ ' for clamped cylindrical shells

\begin{tabular}{|c|c|c|c|c|c|c|c|c|}
\hline \multirow{2}{*}{ Lamination } & \multirow{2}{*}{$\bar{y}$} & \multicolumn{7}{|l|}{$\bar{x}$} \\
\hline & & 0.2 & 0.3 & 0.4 & 0.5 & 0.6 & 0.7 & 0.8 \\
\hline \multirow{7}{*}{$0 / 90 / 0 / 90$} & 0.2 & 71.34 & 78.68 & 88.30 & 90.61 & 88.23 & 78.68 & 71.34 \\
\hline & 0.3 & 71.05 & 78.22 & 87.96 & 92.36 & 87.96 & 78.22 & 71.05 \\
\hline & 0.4 & 70.93 & 78.20 & 88.54 & 95.88 & 88.54 & 78.20 & 70.93 \\
\hline & 0.5 & 7I.II & 78.49 & 89.10 & 100.00 & 89.10 & 78.49 & 71.11 \\
\hline & 0.6 & 70.94 & 78.20 & 88.54 & 95.88 & 88.54 & 78.20 & 70.93 \\
\hline & 0.7 & 71.05 & 78.22 & 87.96 & 92.36 & 87.96 & 78.22 & 71.05 \\
\hline & 0.8 & 71.14 & 78.45 & 88.04 & 90.59 & 88.24 & 78.51 & 71.20 \\
\hline \multirow{7}{*}{0.000494} & 0.2 & 74.09 & 77.65 & 83.40 & 89.83 & 83.42 & 77.69 & 74.15 \\
\hline & 0.3 & 76.29 & 80.57 & 86.83 & 92.59 & 86.84 & 80.62 & 76.36 \\
\hline & 0.4 & 80.18 & 85.32 & 92.00 & 96.93 & 92.00 & 85.35 & 80.23 \\
\hline & 0.5 & 85.27 & 90.33 & 96.25 & 100.00 & 96.25 & 90.33 & 85.27 \\
\hline & 0.6 & 80.23 & 85.36 & 92.00 & 96.93 & 92.00 & 85.32 & 80.18 \\
\hline & 0.7 & 76.36 & 80.62 & 86.84 & 92.59 & 86.83 & 80.57 & 76.29 \\
\hline & 0.8 & 74.15 & 77.69 & 83.41 & 89.82 & 83.40 & 77.64 & 74.09 \\
\hline
\end{tabular}




\section{Conclusion}

Present results are in close agreement with those of the benchmark problems. Thus the finite element code used here is suitable for analyzing free vibration problems of stiffened cylindrical panels with cutouts. The relative free vibration performances of shells are expected to be very useful in decision-making for practicing engineers. The information regarding the behavior of clamped stiffened cylindrical shell with eccentric cutouts for a wide spectrum of eccentricity for cross ply and angle ply shells may also be used as design aids for structural engineers.

\section{Acknowledgements}

None.

\section{Conflict of interest}

The author declares no conflict of interest

\section{References}

1. Markus S. The Mechanics of Vibrations of Cylindrical Shells. UK: Elsevier; 1998.

2. Arnold RN, Warburton GB. Flexural vibrations of the walls of thin cylindrical shell having freely supported ends. Proc $R$ Soc Lond A. 1949;197(1049):238-256.

3. Arnold RN, Warburton GB. The flexural vibration of thin cylinders. Proc Inst Mech Eng. 1953;167:62-80.

4. Warburton GB. Vibration of thin cylindrical shell. J Mech Eng Sci. $1965 ; 7: 399-407$.
5. Leissa W, Lee JK, Wang AJ. Vibrations of cantilevered shallow cylindrical shells of rectangular planform. Journal of Sound and Vibration. 1981;78(3):311-328.

6. Chung H. Free vibration analysis of circular cylindrical shells. Journal of Sound and Vibration. 1981;74(3):331-350.

7. Soldatos KP. Free vibrations of antisymmetric angle-ply laminated circular cylindrical panels. Quarterly Journal of Mechanics and Applied Mathematics. 1983;36(2):207-221.

8. Noor K, Burton WS. Assessment of computational models for multilayered composite shells. Applied Mechanics Review. 1990;43(4):67-97.

9. Leissa W. Vibration of shells. NASA SP-288, 1973, Reprinted by Acoustical Society of America. USA: America Institute of Physics; 1993.

10. Dennis ST, Palazotto AN. Static response of a cylindrical composite panel with cutout using geometrically nonlinear theory. AIAA Journal. 1990;28(6):1082-1088

11. Nanda N, Bandyopadhyay JN. Nonlinear free vibration analysis of laminated composite cylindrical shells with cutouts. Journal of Reinforced Plastics and Composites. 2007;26(14):1413-1427.

12. Vasiliev VV, Jones RM, Man LL. Mechanics of Composite Structures. USA: Taylor and Francis; 1993.

13. Qatu MS. Vibration of Laminated Shells and Plates. UK: Elsevier; 2004.

14. Sahoo S, Chakravorty D. Finite element vibration characteristics of composite hypar shallow shells with various edge supports. Journal of Vibration and Control. 2005;11(10):1291-1309.

15. Chakravorty D, Sinha PK, Bandyopadhyay JN. Applications of FEM on free and forced vibration of laminated shells. ASCE Journal of Engineering Mechanics. 1998;124(1):1-8. 